\title{
A Call for the Structured Physicist Report
}

John M. Boone, PhD

Department of Radiology, University of California, Davis, Sacramento, California.

Mahadevappa Mahesh, MS, PhD

The Johns Hopkins University School of Medicine, Baltimore, Maryland

Eric L. Gingold, PhD

Department of Radiology, Thomas Jefferson University, Philadelphia, Pennsylvania.

J. Anthony Seibert, PhD

Department of Radiology, University of California, Davis, Sacramento, California

Follow this and additional works at: https://jdc.jefferson.edu/radiologyfp

Part of the Radiology Commons

Let us know how access to this document benefits you

\section{Recommended Citation}

Boone, PhD, John M.; Mahesh, MS, PhD, Mahadevappa; Gingold, PhD, Eric L.; and Seibert, PhD, J. Anthony, "A Call for the Structured Physicist Report" (2016). Department of Radiology Faculty

Papers. Paper 41.

https://jdc.jefferson.edu/radiologyfp/41

This Article is brought to you for free and open access by the Jefferson Digital Commons. The Jefferson Digital Commons is a service of Thomas Jefferson University's Center for Teaching and Learning (CTL). The Commons is a showcase for Jefferson books and journals, peer-reviewed scholarly publications, unique historical collections from the University archives, and teaching tools. The Jefferson Digital Commons allows researchers and interested readers anywhere in the world to learn about and keep up to date with Jefferson scholarship. This article has been accepted for inclusion in Department of Radiology Faculty Papers by an authorized administrator of the Jefferson Digital Commons. For more information, please contact: JeffersonDigitalCommons@jefferson.edu. 


\title{
As submitted to:
}

\section{Journal of the American College of Radiology}

\author{
And later published as:
}

\section{A Call for the Structured Physicist Report}

\author{
Volume 13, Issue 3, March 2016, pp. 307-309
}

DOI: http://dx.doi.org/10.1016/j.jacr.2015.12.016

John M. Boone, PhD, Mahadevappa Mahesh, MS, PhD, Eric L. Gingold, PhD, J. Anthony Seibert, $\mathrm{PhD}$

\section{INTRODUCTION}

The field of diagnostic radiology continues to struggle with the clinical adoption of the structured interpretive report, with many radiologists preferring a semistructured, free-text dictation style to a more rigid, highly structured approach that some professional leaders have promoted [1]. Although structured reporting compliance in the radiologist community has been difficult to achieve, diagnostic radiologists have been thinking about and discussing this important issue for many years; it is also a part of the ACR's Imaging 3.0_ campaign [2]. In the breast imaging community, the wellestablished BI-RADS_ recommendations produce a very structured report, with a discussion of interpretive findings culminating in a numeric BI-RADS score ranging from 0 to 6 [3]. Unlike some interpretive radiology reports, which can be ambiguous in terms of the next course of action, the BIRADS scale is not only a diagnostic scale but also prescriptive of what the necessary follow-up should be.

The diagnostic medical physics community needs to strongly consider adopting a structured physicist report (SPR). In this article, we discuss the motivation and potential benefits of developing the SPR. Although the SPR can apply to any modality, we propose to begin with CT, and we focus on CT in this proposal of a conceptual framework, then taking lessons learned from this exercise to extend the SPR to other diagnostic imaging modalities.

The benefits of the SPR are many. The consumers of these reports are typically radiology or hospital administrators, regulators, surveyors, and sometimes radiologists. A concise and relatively uniform SPR inCTwill allow readers who are not well versed in radiologic physics to more easily make sense of these reports. When different imaging centers within a network under central administrative control receive reports that may be produced by different physicists or different physics consulting groups, a common report format will make the report interpretation much more straightforward. Inspectors from regulatory agencies and accreditation bodies will have an easier job understanding the physicists' work, if standardized using the same general template across sites. In addition, the use of a standard format (and form) for the SPR in CT would enable the development of a broad-scale data registry for both dose and image quality metrics. 


\section{DETAILS OF THE SPR}

We propose that the SPR for CT should be a spreadsheet (eg, a Microsoft Excel workbook), with the necessary tabs (worksheets) in place to cover routine CTscanner performance tests. The first tab should contain simple identifiers, such as room number institution, date of measurement and report date; medical physicist's name, contact information, board certification type and date, and signature; and the details of the scanner type, model, serial number, usage, and so on. The second tab should be the executive summary, consisting of two or more paragraphs that describe the overall summary of the evaluation. Subsequent tabs should be standardized in format and order and contain the typical performance parameters that are evaluated in CT regularly, including radiation dose measurements (volumetric CT dose index [CTDIvol]), assessment of dose display accuracy, spatial resolution, contrast resolution, CT number accuracy and uniformity, etc. Imaging performance metrics might include slice sensitivity profile, contrast-to-noise ratio, modulation transfer functions, 3-D noise power spectrum, and so on, measured in commonly available phantoms. In addition, optional (but standardized) tabs of the CT SPR would address other parameters that are pertinent to the specific clinical utilization of a given CT scanner. For example, CT scanners that are used routinely for pediatric scanning should have more detailed assessments of image quality and dose measurements for a range of pediatric CT examination types and patient (phantom) sizes. CT systems that are used for biopsy procedures should also have detailed scatter-field measurements in the scanner room, along with assessment of temporal CT imaging performance, etc. Other CT specialty applications such as cardiac imaging, perfusion scanning, shuttlemode scanning, dualenergy scanning, and so forth, can and should be reported in optional tabs, designed to address the image quality and dose performance of these specialized and often optional features of specific CT scanners. The use of spreadsheetbased reports is already common practice in the diagnostic imaging physics community; this proposal is merely to produce a more structured (and standardized) template and to use these structured reports as the input into a national database.

\section{A NATIONAL DATABASE OF CT SCANNER PERFORMANCE}

Unlike most radiologist-generated reports for clinical patient image interpretation, the annual physicist report for CT systems contains mostly quantitative values that are measured or otherwise assessed during scanner evaluation: dose measurements such as the CT dose index (CTDI-100), spatial resolution, contrast resolution, noise assessment, CT number accuracy, and other parameters that describe CT scanner quantitative performance at a technical level. Therefore, these standardized report templates can be used as a mechanism to create a national (or international) database on CT scanner performance, similar to the data generated by the National Examination of X-Ray Trends surveys [4] that were performed in the past by the Center for Devices and Radiological Health (formerly the Bureau of Radiological Health) within the FDA. The SPR format for CTis proposed to be defined by a task group of the American Association of Physicists in Medicine (AAPM). The task group's charge is to define and create a report divided into sections (tabs) with human-readable data, including individual parameter values, tables of values, appropriate graphs of data and data trends, and in some cases free text that describes and interprets the associated numeric data. Data for a national CT registry would be linked to a special registry tab at the end of the report, with dense quantitative information not necessarily designed for human readability. Instead, this tab would collate all of the useful quantitative measurement data from the rest of the report in specific, machine-readable fields performed automatically in spreadsheet software by linking cells across tabs. 
The data in this tab could be exported and uploaded to an AAPM-hosted website to be stored, optionally deidentified, and exported to the national registry (yet to be established).

Groups working within the AAPM structure, with the major CT vendors, have published CT protocols (by vendor), that are available to anyone through the AAPM's website. These protocols have been developed for routine head, chest, and abdominal and pelvic CT, along with CT brain perfusion studies, low-dose lung cancer screening CT protocols, and others [5]. Although these protocols are freely available, they do not represent a CT dose registry, per se.

The ACRDose Index Registry_ is a national registry that is designed to record and tabulate dose indices pertinent to clinical CT examinations such as head CT, abdominal and pelvic CT, and so on [6]. The Dose Index Registry program is designed to evaluate how CT scanners are used clinically for a range of most commonly used CT protocols. The SPR for CT, the topic of this column, would not necessarily report the dose information of clinical protocols but rather would focus on the output of the CT scanner characterized using normalized metrics such as CTDI- 100 (peripheral), CTDI-100 (center), and CTDI-100 (air), in units of milligrays per 100 milliampereseconds over the range of other parameters such as x-ray tube potential, field of view (ie, bowtie filter), phantom size $(16$ or $32 \mathrm{~cm}$ ), etc. These are just a few examples of the parameters that are most important when performing consistency testing of CT scanner hardware. The methodologies for evaluating these metrics are already quite standardized, so the data should require little if any further processing to be useful for benchmarking.

\section{NATIONAL DATABASE USAGE}

After the collection of sufficient numbers of CT SPRs, the AAPM CT scanner performance registry would be able to describe the performance of a large number of CT scanner models using data-rich statistical methods, such as the median (mean), range (standard deviation), and cumulative distribution (or histogram) of specific performance metrics (eg, CTDIvol in milligrays per 100 milliampere-seconds for a 16-cmdiameter phantomat $120 \mathrm{kV}$ ) on each model and make of CT scanner that is in current use across the United States. As the popularity of the registry grows, it will become an important tool to provide normative data for individual CT scanner models. For example, after measuring a specific CTDIvol (eg, small scan field of view, $16 \mathrm{~cm}$ poly(methyl methacrylate) phantom, and $120 \mathrm{kV}$ ) for a given CT scanner, comparing the value against the statistical distribution allows a physicist to objectively assess deviation from the median performance level, which at the physicist level constitutes evidence-based medicine. It also makes consistency testing data driven. These statistical data would also provide solid quantitative assessment for determining when a scanner is performing nominally andwhen it should be considered out of compliance and in need of service, x-ray tube replacement, or decommissioning. This information would be helpful for justifying equipment replacement, another potential benefit of this project.

\section{SUMMARY}

We believe that the SPR is an important and useful concept, which will result in a uniform report format for efficiently conveying the results of periodic compliance testing of diagnostic imaging systems. The proposal is to initiate the SPR concept using CT annual reports, given the complexity of these systems and the quantitative nature of their output metrics for both dose and image quality. A significant benefit of developing a standard reporting format is to develop a large database of CT 
scanner performance. This will allow CT scanner performance evaluation, across myriad performance parameters encompassing both dose and image quality, to be data driven and conducted in the context of specificCTscannermakes andmodels. This program should provide clinical medical physicists with new tools to augment their practice and increase the value of their product in their clinical settings, be it in a consulting, hospital, or university medical center capacity. Additionally, the effort could represent a framework for future SPRdocuments within radiology and the health care enterprise.

\section{REFERENCES}

1. Langlotz CP. The radiology report: a guide to thoughtful communication for radiologists and other medical professionals. CreateSpace Independent Publishing Platform. November 2015. ISBN: 978-1515174080.

2. American College of Radiology. ACR Imaging 3.0_.Available at: http://www.acr.org/Advocacy/ Economics-Health-Policy/Imaging-3. Accessed November 19, 2015.

3. American College of Radiology. ACR BIRADS_. Available at: http://www.acr.org/QualitySafety/Resources/BIRADS. Accessed November 19, 2015.

4. 4. US Food and Drug Administration. Nationwide Evaluation of X-Ray Trends (NEXT). Available at: http://www.fda.gov/RadiationEmittingProducts/RadiationSafety/NationwideEvaluationofXRay'TrendsNEXT/default.htm. Accessed November 25, 2015.

5. American Association of Physicists in Medicine. Computed tomography protocols by the American Association of Physicists in Medicine.Available at: http://www.aapm.org/pubs/CTProtocols/. Accessed November 19, 2015.

6. American College of Radiology. American College of Radiology Dose Index Registry core documents. Available at: http://www.acr.org/w/media/ACR/Documents/PDF/QualitySafety/NRDR/DIR. Accessed November 19, 2015.

John M. Boone, PhD, and J. Anthony Seibert, PhD, are from the Department of Radiology, University of California, Davis, Sacramento, California. Mahadevappa Mahesh, MS, PhD, is from The Russell H. Morgan Department of Radiology and Radiological Science, The Johns Hopkins University School of Medicine, Baltimore, Maryland. Eric L. Gingold, PhD, is from the Department of Radiology, Thomas Jefferson University, Philadelphia, Pennsylvania.

The authors have no conflicts of interest related to the material discussed in this article.John M. Boone, PhD: Department of Radiology, University of California, Davis, Medical Center, Suite 3100, Ellison Building, 4860 Y Street, Sacramento, CA 95817; e-mail: jmboone@ucdavis.edu. 